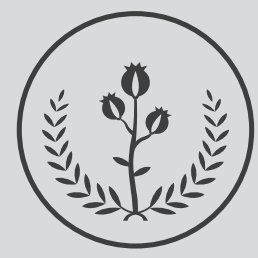

FUCS $-x$

$N^{0} 1.2021$

\title{
Conocimiento y capacitación virtual de la responsabilidad del enfermero del quirófano/ instrumentador quirúrgico
}

\section{Virtual intervention and training on operating room nurses/surgical instrument technicians responsibility}

${ }^{a}$ Facultad de Instrumentación Quirúrgica, Fundación Universitaria de Ciencias de la Salud. Bogotá DC, Colombia, abogado especialista en derecho laboral y seguridad social.

\section{R E S U M E N}

Objetivo: realizar el diagnóstico y capacitación del enfermero de quirófano/instrumentador quirúrgico (EQ/IQ) frente a la responsabilidad médica y legal como eje de la seguridad del paciente. Métodos: estudio en dos fases, la primera corresponde a un análisis descriptivo de corte trasversal con la participación de $23 \mathrm{EQ} / \mathrm{IQ}$; la fase II es un estudio cuasi experimental con $15 \mathrm{EQ} / \mathrm{IQ}$. Las pruebas pre y postest (aplicación de un OVA) permitieron describir la efectividad de la intervención. Resultados: la fase I, prueba piloto, evidenció la falta de conocimiento en responsabilidad legal y médica. La fase II mostró un cambio significativo después de la intervención con el OVA; la capacitación en temas básicos de responsabilidad legal y médica fue satisfactoria y se identificó el cambio posintervención. Discusión: los resultados y la literatura científica permiten establecer que existe un desconocimiento en responsabilidad médica y su influencia en la seguridad del paciente. Conclusión: la capacitación se convierte en un aporte para la garantía de calidad en la prestación de servicios de salud.

Palabras clave: enfermería de quirófano, instrumentador quirúrgico, responsabilidad médica, iatrogenia, objeto virtual de aprendizaje (OVA), seguridad del paciente.

Historia del artículo:

Fecha recibido: octubre 21 de 2019

Fecha aceptado: noviembre 5 de 2019
Autor para correspondencia. Sr. Henry David Tole Acosta hdtole@fucsalud.edu.co
DOI

10.31260/RepertMedCir.01217273.949 


\section{A B S T R ACT}

Introduction: to assess and identify operating room nurses/surgical instrument technicians (SN/SIT) knowledge on legal and medical responsibility and train them on this topic as the essential aspect for ensuring patient safety. Methods: a two-phase study was carried out, the first phase consisted of a cross sectional descriptive analysis including 23 SN/SIT; phase II was a quasiexperimental intervention study including 15 SN/SIT. The pre and post Open Virtual Application [OVA] intervention results enabled evaluating its efficacy. Results: In Phase I a pilot test evidenced lack of knowledge on legal and medical responsibility. Phase II showed a significant change after OVA intervention; training on basic topics on legal and medical responsibility was satisfactory and a post-intervention change was identified. Discussion: the study results and the scientific literature evidenced there is a lack of understanding on legal and medical responsibility and how it affects patient safety. Thus, training contributes to guarantee quality health care services.

Key words: higher education, profession; cytotechnology; knowledge; cytology; immunohistochemistry; histological technique.

\section{INTRODUCCIÓN}

El enfermero de quirófano/instrumentador quirúrgico $(\mathrm{EQ} / \mathrm{IQ})$ es un trabajador del área de la salud que realiza funciones prácticas en el ámbito quirúrgico, hace asistencia directa en las especialidades médicoquirúrgicas y verifica gestión y administración de centrales de esterilización y área quirúrgica. En Colombia y algunos países de latinoamérica el desarrollo de la profesión se enmarca en el perfil de la instrumentación quirúrgica, reglamentada en Colombia por la ley 784 del 2002, en donde refiere que el "ejercicio requiere título de idoneidad universitaria, basada en una formación científica, técnica y humanística, docente e investigativa y cuya función es la planeación organización, dirección, ejecución supervisión y evolución de las actividades que competen al enfermero de quirófano/ instrumentador quirúrgico como parte integral del equipo de salud". ${ }^{1}$

En el saber del profesional sanitario la responsabilidad del actuar en salud trata de mitigar al mínimo los eventos adversos y resguardar la seguridad del paciente, al disminuir las iatrogenias y generar un enfoque de la responsabilidad médica. Una de las competencias del (EQ/IQ) es tener un grado de conocimiento en los temas de responsabilidad legal y médica, a partir del diligenciamiento y la disposición de los documentos medico legales, ${ }^{2}$ ya que estos conceptos se relacionan con el actuar diario y la toma de decisiones autónomas en la profesión. ${ }^{1}$ Por esta razón el registro oportuno del consentimiento informado, lista de chequeo y recuento de instrumental quirúrgico, se convierten en requisitos esenciales en el acto quirúrgico y en la seguridad de paciente. ${ }^{3}$

De acuerdo con lo anterior, el (EQ/IQ) debe ejercer la profesión con estricto cumplimiento de los parámetros de calidad. ${ }^{4}$ El IBEAS, ${ }^{5}$ describe los eventos adversos más frecuentes como cirugía en el lado equivocado, retención de cuerpo extraño como compresas, gasas o instrumentos, errores de transfusión, eventos relacionados con la anestesia, lesiones causadas por el equipo quirúrgico y errores en la medicación; ${ }^{6}$ los dos primeros competen al (EQ/IQ). Por otro lado, la incidencia de cuerpos extraños olvidados en cavidad la cual es entre 1 por cada 100 y hasta 1 por cada 10.000 casos intervenidos ${ }^{7}$ conllevan sucesos iatrogénicos, aumentando la morbimortalidad, sobrecostos a la institución y posibles problemas médicolegales. ${ }^{8}$ El error del sitio operatorio representa entre 1,7 y el $7 \%$ de las demandas por mala praxis y recae la responsabilidad principalmente en el cirujano, sin embargo, los estudios incluyen el equipo quirúrgico. ${ }^{9}$

Por consiguiente, el grupo investigador buscó identificar el nivel de conocimiento en conceptos básicos sobre responsabilidad médica, legal y civil $^{10}$, mediante el diagnóstico de saberes del (EQ/IQ) considerando los conocimientos en el ámbito de la responsabilidad en pro de la seguridad del paciente. La intervención fue una capacitación y evaluación de un objeto virtual de aprendizaje (OVA) como estrategia educativa y de mejora de conocimientos en la práctica médica, ${ }^{11,12}$ la elección de esta modalidad permitió que los participantes accedieran a la información y al aprendizaje en forma autónoma ${ }^{13}$ con una actitud autorregulada en el proceso formativo. ${ }^{14,15}$

\section{MÉTODOS}

Diseño. Primera fase: estudio descriptivo de corte trasversal sobre los conocimientos en responsabilidades civil y médicas básicas. Los resultados obtenidos fueron insumo para diseñar el OVA en aspectos básicos de responsabilidad en el campo del EQ/IQ . Segunda fase: se realizó un estudio cuasiexperimental, pre y postest respecto a la implementación de un curso de tipo OVA con aspectos básicos de responsabilidad en el campo del EQ/IQ. 
Participantes. Primera fase: participaron de manera voluntaria $\mathrm{n}=23$ profesionales de instrumentación quirúrgica/ enfermero de quirófano, que laboraban en salas de cirugía de una institución hospitalaria de Bogotá. Segunda fase: de manera voluntaria incluyó $\mathrm{n}=15$ instrumentadores profesionales, que tuviesen habilidades básicas en el manejo de herramientas virtuales y que se desempeñaran directamente en el quirófano.

Procedimiento: Primera fase: se elaboró un instrumento piloto con 18 preguntas de responsabilidad civil, médica y legal para ajustar el cuestionario final y así determinar las temáticas del diseño del OVA. Segunda fase: pre y postest, aplicando el cuestionario adaptado de la primera fase (OVA).

Intervención. Se diseñó y aplicó un OVA que versa sobre aspectos básicos de la responsabilidad en el campo del EQ/ IQ. Consta de 5 temas: IQ vs responsabilidad, documentos legales del IQ, evento adverso y responsabilidad, conductas probables de daño en el paciente de cirugía y protocolos y estándares nacionales e internacionales de seguridad del paciente. La duración fue de un mes y medio para desarrollar competencias sobre la temática.

Instrumento. Se ajustó el instrumento piloto para realizar la evaluación pre y postest con 12 preguntas y respuesta dicotómica (si/no), para determinar los conocimientos previos y posteriores a la intervención.

Consideraciones éticas. Se tuvo en cuenta la normativa internacional y nacional, el proyecto fue aprobado por el comité de ética de investigación en seres humanos del Hospital de San José - FUCS.

\section{RESULTADOS}

Primera fase: se realizó un estudio de corte trasversal con $n=23$ participantes. Se aplicó una prueba piloto de 18 preguntas que evaluó los conocimientos básicos de responsabilidad civil y médica. La población se caracterizó por ser profesionales con escolaridad de pregrado, diplomado y posgrado, en la prueba piloto los resultados se describieron en un plano factorial, de correspondencias múltiples (figura 1). La prueba piloto mostró el bajo conocimiento de los participantes frente a la responsabilidad legal y temas de legislación médica. Se identifica la relación de los niveles de escolaridad con el de conocimiento, hallando que no hay relación estadística significativa con las respuestas efectuadas. Los temas con el más bajo nivel de conocimientos son imprudencia, evento adverso, impericia y negligencia. Además, no hay una relación directa entre el nivel de formación y el tiempo de experiencia laboral.

Segunda fase. Se realizó un estudio cuasi experimental pre y postest respecto a la implementación de un OVA (curso de aspectos básicos de responsabilidad). Participaron $\mathrm{n}=17 \mathrm{EQ} / \mathrm{IQ}$ (tabla 1) que laboraban en el área asistencial (quirófano) en instituciones de servicios de salud en la ciudad de Bogotá; se excluyeron 2 que no continuaron en el estudio.

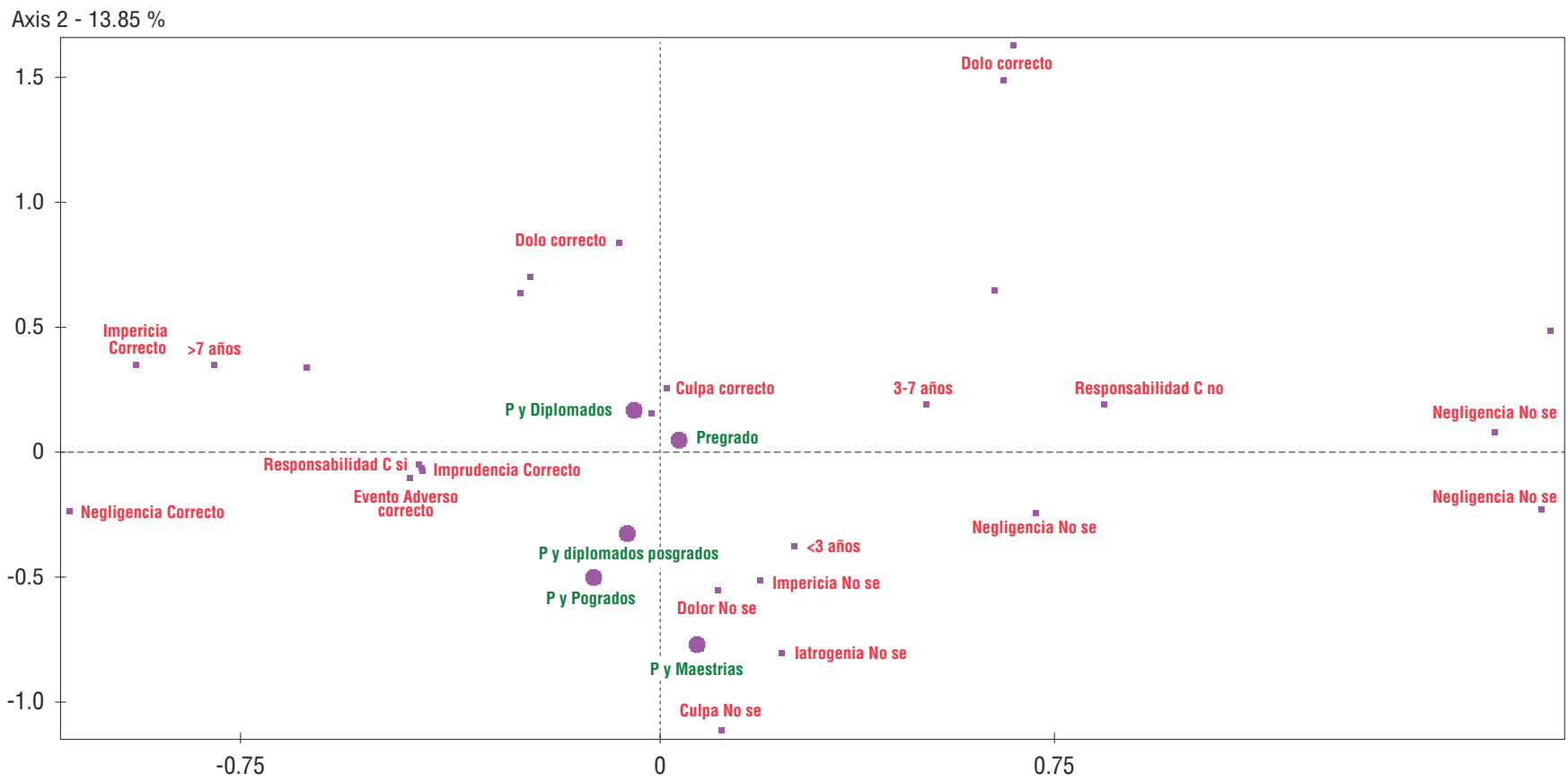

Figura 1. Nivel de conocimientos en el pre y postest. Fuente: el autor. 
Tabla 1. Características socio demográficas

\begin{tabular}{|c|c|c|c|}
\hline Edad & Prom (de) $27,7(7,79)$ & Edad & Prom (de) 27,7 (7,79) \\
\hline Sexo & $\mathbf{n}(\%)$ & Experiencia profesional & $\mathbf{n}(\%)$ \\
femenino & $12(80)$ & menos de 1 año & $7(46,6)$ \\
masculino & $3(20)$ & $1-4$ años & $1(6,6)$ \\
\cline { 1 - 2 } Escolaridad & $\mathbf{n}(\%)$ & $5-7$ años & $3(20)$ \\
pregrado & $12(80)$ & Más 7 años & $4(26,6)$ \\
\hline
\end{tabular}

Fuente: el autor.

Respecto al nivel de conocimientos identificado en los participantes en el pre y el postest, se observa en la (figura 1) que previo a la intervención con el OVA el nivel era deficiente $(=/<2$. 0$)$ a diferencia del postest, que muestra una mejora en el nivel de conocimientos $(=/>4.0)$ (figura 2).

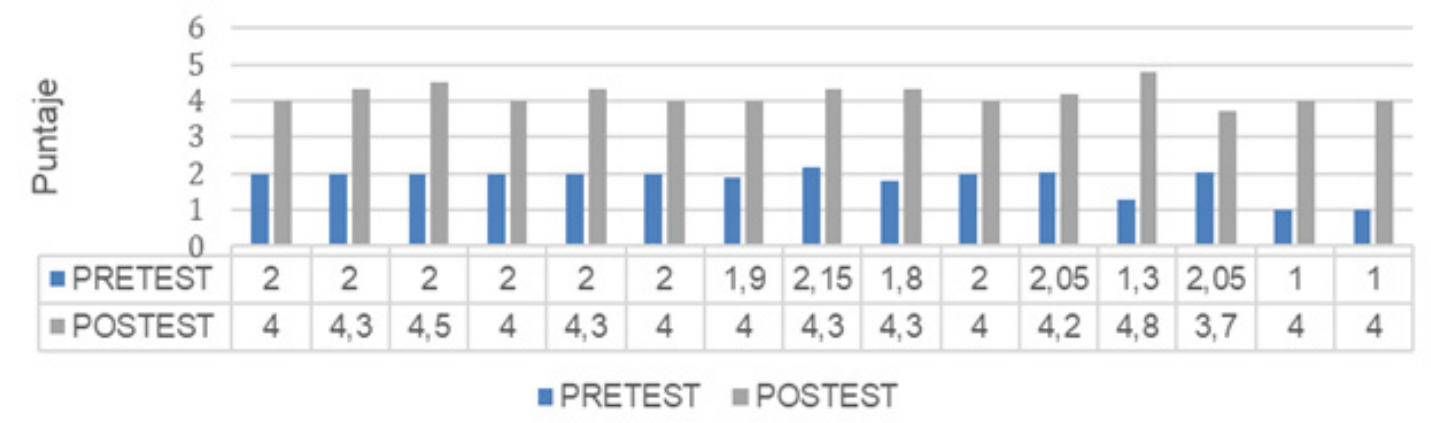

Figura 2. Puntaje pre y postest. Fuente: el autor.

\section{DISCUSIÓN}

Los resultados de la fase I del estudio permitieron consolidar el instrumento de evaluación de responsabilidad civil y médica, así como identificar las pautas para el diseño del OVA. Margain y col. ${ }^{15}$ sugieren la importancia de reconocer las necesidades formativas de una población para diseñar un OVA. En el ámbito educativo del área de la salud se deben implementar metodologías y estrategias didácticas que permitan la consolidación del aprendizaje según Yánez y col. ${ }^{16}$ La prueba piloto mostró el bajo conocimiento sobre la responsabilidad legal y legislación médica de los participantes. Es así como la OMS plantea que gran parte de los prejuicios y temores del personal de salud se generan por la ausencia de una cultura de seguridad del paciente y por la omisión del sistema de salud al reconocer los errores cometidos. ${ }^{17}$ Grunfeld ${ }^{18}$ plantea la importancia del grado de formación de los profesionales de la salud en cuanto a los temas relacionados con error médico y práctica segura. Por otro lado, el bajo nivel obtenido en el nivel de conocimientos en imprudencia, evento adverso, impericia y negligencia, muestra la relación entre el nivel de formación y el tiempo de experiencia laboral. Tachiquín-Sandoval y col. ${ }^{19}$ plantean la necesidad de hacer un llamado a las instancias educativas para incluir el tema de responsabilidad legal médica en los programas curriculares o incluir una inducción sobre este tema para consolidar los conceptos y las consecuencias de una mala praxis. Recomiendan considerar el desinterés de este personal de salud dado por la demanda de trabajo y la experiencia laboral. De esta manera, este resultado muestra la importancia de que la responsabilidad legal médica está inmersa en los currículos de los programas del área de la salud, fortaleciendo el perfil de egreso de los futuros profesionales.

En la fase II cuasi experimental, el pretest es deficiente $(=/<2.0)$ respecto al resultado postest $(=/>4.0)$, lo cual revela una mejora en el nivel de conocimientos de responsabilidad legal y médica. Bernal y Botero ${ }^{20}$ hallaron en su estudio una confusión de conocimientos en los profesionales de la salud en los conceptos básicos de responsabilidad médica, como el error médico y el evento adverso. Rivera ${ }^{21}$ plantea que la mayoría de las reclamaciones por responsabilidad médica se dan por el inadecuado manejo de algunos aspectos del acto médico, por lo que es necesario la introducción en el currículo de temas como: lex artis, historia clínica, consentimiento médico y comunicación con los pacientes, al ser parámetros de seguridad. Machado y col. ${ }^{22}$ hacen referencia al escaso conocimiento y comprensión de las iatrogenias, los errores médicos y las consecuencias generadas por una mala práctica. Carvalho y Ceolim ${ }^{23}$ muestran como el bajo nivel de conocimientos e información respecto a la iatrogenia tiene relación directa con la seguridad del paciente. Monteiro y $\mathrm{col} .{ }^{24}$ realizan una reflexión respecto al actuar y la conciencia quirúrgica inmersa dentro de las actividades en el quirófano y que integran la responsabilidad médica. Blomberg y col. ${ }^{25}$ realizan un aporte significativo de la responsabilidad desde 
lo moral hasta el valor ético personal del profesional de salud. Para los autores los conocimientos en responsabilidad civil y médica son fundamentales en la práctica profesional de la enfermería de quirófano/instrumentación quirúrgica o en cualquier profesión en salud, puesto que el conocimiento teórico es la base para comprender los términos y las consecuencias de la iatrogenia, daño, dolo, eventos adversos y documentos legales en la atención del paciente. En el caso del EQ/IQ se demuestra la necesidad de implementar espacios académicos y prácticos de formación en aspectos de responsabilidad legal y médica, para incrementar practicas seguras en la atención en salud.

Por otro lado, la implementación del OVA es una metodología de aprendizaje que permite el acceso a la información, la autonomía y autorregulación del estudiante. $^{26}$ Los resultados postest muestran un avance significativo en el nivel de conocimiento de responsabilidad médica y la efectividad del OVA como herramienta didáctica en el personal de salud. Esto demuestra que las tecnologías de la información y la comunicación TICS se integran de manera efectiva a los procesos educativos de la era contemporánea. ${ }^{27,28}$ Forero y col. ${ }^{29}$ sugieren la implementación de los OVA como estrategia de enseñanza y aprendizaje, complementaria al ser una herramienta de utilización en el tiempo libre, optimizándolo y facilitando la interdisciplinariedad.

Desde el punto de vista del autor se integra la implementación del OVA con el aporte del EQ/IQ desde los conceptos en responsabilidad médica hasta el mejoramiento de prácticas seguras en la atención de pacientes, a través de la formación de un grupo quirúrgico en estos temas. Esto demuestra la necesidad formativa de los EQ/IQ y demás profesionales de la salud en temas transversales que se integran al saber profesional, con metodologías de fácil acceso para la capacitación, teniendo en cuenta la alta carga laboral que puede tener este tipo de personal.

En cuanto a las edades de los participantes del estudio (20-25 años), se obtuvo una mayor calificación postest en los participantes de menor edad a diferencia de las personas mayores, esto demuestra la facilidad de acceso a estas herramientas en las generaciones más jóvenes. ${ }^{30}$ Este resultado permite reconocer la importancia de incentivar y motivar a los profesionales del área de la salud de todas las edades al uso de herramientas tecnológicas y a hacer conciente la importancia de la educación posgradual, como parte de la consolidación de los conocimientos en pro de la seguridad del paciente y mejor praxis. El autor concluye que la seguridad del paciente es un eje transversal en salud que se fortalece con la práctica y por tanto, los temas de índole legal y normativo y en la formación hacen parte de este tópico evidenciando la necesidad constante de generar estos programas formativos en el currículo, para disminuir los requerimientos jurídicos por falta de manejo de estos conceptos.

\section{RECOMENDACIONES Y LIMITACIONES}

El tema de responsabilidad civil y médica es poco abarcado en el área de la salud, resultando pocos estudios al respecto, debido talvez a la limitación de tiempo relacionada con la carga laboral. Sin embargo, se debe considerar la posibilidad de incluir temáticas formativas que puedan generar impacto en la práctica del profesional de la salud. Se sugiere continuar con estudios similares que incluyan mayor población e interdisciplinariedad para establecer diferencias y similitudes en la responsabilidad médica de acuerdo con el ámbito de cada profesional.

\section{CONFLICTO DE INTERESES}

El autor declara no tener ningún conflicto de intereses.

\section{REFEREN CIAS}

1. Asociación Colombiana de Instrumentadores Quirúrgicos Profesionales, Asociación Colombiana de Facultades de Instrumentación Quirúrgica, Colegio Colombiano de Instrumentación Quirúrgica. Perfil y competencias del profesional en instrumentación quirúrgica en Colombia. Colombia2014. p. 52.

2. Tole HD, Cadavid N. Instrumentador quirúrgico: Iatrogenia, eventos frecuentes y responsabilidad en el quirófano. Repert Med Cir. 2018;27(2):124-30.

3. Escobar López MT, Novoa Torres E. Análisis de formatos de consentimiento informado en Colombia. Problemas ético-legales y dificultades en el lenguaje. Rev latinoam bioet. 2016;16(30-31):147.

4. Quinteros P. El compromiso del instrumentista quirúrgico con el paciente y el equipo tratante. Medwave. 2004;4(9):e2671.

5. Organización Mundial de la Salud. IBEAS: red pionera en la seguridad del paciente en Latinoamérica. Hacia una atención hospitalaria más segura. Organización Mundial de la Salud; 2010. p. 16.

6. Tole Acosta HD. Panorama latinoamericano del cuerpo extraño olvidado en el interior del paciente, desde la vista del instrumentador quirúrgico. Rev CSV. 2015;7(2):42-52.

7. Chinelli J, Olivera E, Rodríguez G. Oblitos en Cirugía. Análisis de casuística y estrategias para su prevención. Anfamed. 2017;4(2):21 38.

8. Alfonso Borráez O, Alfonso Borráez B, Orozco M, Matzalik G. Cuerpos extraños en abdomen: presentación de casos y revisión bibliográfica. Rev Colomb Cir. 2009;24(2):114-22.

9. García-Germán Vázquez D, Sanz Martín J, Canillas de Rey F. Cirugía en sitio erróneo en cirugía ortopédica y traumatología. Estudio 
de sentencias judiciales y reclamaciones Trauma (Majadahonda) 2010;21(4):256-61.

10. Tole Acosta HD. Diagnóstico y evaluación de la responsabilidad civil y médica del profesional de instrumentación quirurgica en el ejercicio de la profesión del hospital de san José. Bogotá: Fundación Universitaria de Ciencias de la Salud; 2014.

11. Rodríguez Almada H. Los aspectos críticos de la responsabilidad médica y su prevención. Rev Med Uruguay 2001;17(7):17-23.

12. Salas Perea RS. La calidad en el desarrollo profesional: avances y desafíos. Educ Med Super 2000;14(2):136-47.

13. Morales Martín LY, Gutiérrez Mendoza L, Ariza Nieves LM. Guía para el diseño de objetos virtuales de aprendizaje (OVA). Aplicación al proceso enseñanza-aprendizaje del área bajo la curva de cálculo integral. Revista Científica General José María Córdova. 2016;14(18):127-47.

14. Gibelli TI, Chiecher AC. Autorregulación del aprendizaje en entornos mediados por TIC. Una propuesta de intervención en matemática universitaria de primer año. XVIII Congreso Argentino de Ciencias de la Computación. Argentina: Red de Universidades con Carreras en Informática (RedUNCI). p. 10.

15. Margain Fuentes MdLY, Muñoz Arteaga J, Álvarez Rodríguez FJ. Metodología de Aprendizaje Colaborativo fundamentada en patrones para la producción y uso de Objetos de Aprendizaje. Investigación y Ciencia. 2009;17(44):22-8.

16. Yánez ÁC, Silvana Ortiz L, Espinosa VE. Las tecnologías de la comunicación e información (TIC) en salud: un modelo para aplicar en la carrera de Enfermería. Rev iberoam educ investi Enferm. 2016;6(2):29-36.

17. Fernández Cantón SB. El error médico como causa de muerte. Boletín CONAMED-OPS; 2016. p. 26-31.

18. Grunfeld Baeza MV. Los errores médicos en la atención primaria de salud [Internet]. Sociedad Iberoamericana de Información Científica; 2018 [citado 2019 febrero 25]; Recuperado de: https://www.siicsalud.com/acise_viaje/ensiicas-profundo. php?id=156788.

19. Tachiquín-Sandoval R, Romero-Escobedo AC, Padilla-Villalobos LA. Conocimiento sobre responsabilidad profesional en la atención médica ¿falta de actitud, interés o educación? Revista CONAMED. 2014;19(1):23-31.
20. Bernal Moreno EV, Botero Mejía P. Conocimientos y prácticas sobre error médico en profesionales de la salud, de Jaibaná IPS. Primer trimestre 2016.[Tesis] Pereira: Fundación del Área Andina; 2016.

21. Rivera Alvarado MI. ¿Qué se entiende por responsabilidad del acto médico? una reflexión desde la biojurídica. Escritos - Fac Filos. 2014;22(48):123-42.

22. Duarte Sda C, Stipp MA, da Silva MM, de Oliveira FT. Adverse events and safety in nursing care. Revista brasileira de enfermagem. 2015;68(1):136-46, 44-54. Epub 2015/05/07.

23. dos Santos JC, Ceolim MF. [Nursing iatrogenic events in hospitalized elderly patients]. Revista da Escola de Enfermagem da U S P. 2009;43(4):810-7. Epub 2010/01/21. Iatrogenias de enfermagem em pacientes idosos hospitalizados.

24. Monteiro Lima A, Silva Sousa C, Silva Mirancos da Cunha AL. Patient safety and preparation of the operating room: reflection study. J Nurs. 2013;7(1):289-94.

25. Blomberg AC, Bisholt B, Lindwall L. Responsibility for patient care in perioperative practice. Nursing open. 2018;5(3):414-21. Epub 2018/08/01.

26. García Martín M. La autorregulación académica como varaible explicativa de los procesos de parendizaje universitario. Revista de Currículum y Formación de Profesorado. 2012;16(1):203-21.

27. Cabrera Medina JM, Sánchez Medina II, Rojas Rojas F. Uso de objetos virtuales de aprendizaje OVAS como estrategia de enseñanza - aprendizaje inclusivo y complementario a los cursos teóricos - prácticos. Una experiencia con estudiantes del curso físico de ondas. Revista Educación en Ingeniería. 2016;11(22):412.

28. Sierra Varón CA. La educación virtual como favorecedora del aprendizaje autónomo. Panorama. 2011;9:75-87.

29. Fajardo Forero LF, Sotelo Díaz MÁ, Moreno Vela FJ. El uso de los ovas como estrategia de enseñanza-aprendizaje bajo un esquema de educación bimodal. Bogotá2012. p. 14.

30. Gutiérrez Mendoza L, Buitrago A. MR, Ariza N LM. Diseño de un OVA como mediador pedagógico para la enseñanza de la derivada. Bogotá2015. 